\section{Subcellular imaging of epithelium with time-lapse optical coherence tomography}

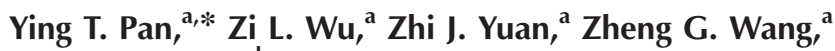 \\ and Cong W. Du

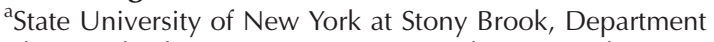 \\ of Biomedical Engineering, Stony Brook, New York \\ 11794 \\ ${ }^{\mathrm{b}}$ State University of New York at Stony Brook, Department \\ of Anesthesiology and Medical Department, \\ Brookhaven National Laboratory, Upton, New York 11973
}

Abstract. We present the first experimental result of direct delineation of the nuclei of living rat bladder epithelium with ultrahigh-resolution optical coherence tomography (uOCT). We demonstrate that the cellular details embedded in the speckle noise in a uOCT image can be uncovered by time-lapse frame averaging that takes advantage of the micromotion in living biological tissue. The uOCT measurement of the nuclear size $(7.9 \pm 1.4 \mu \mathrm{m})$ closely matches the histological evaluation $(7.2 \pm 0.8 \mu \mathrm{m})$. Unlike optical coherence microscopy (OCM), which requires a sophisticated high-NA microscopic objective, this approach uses a commercial-grade single achromatic lens $(\mathrm{f} / 10 \mathrm{~mm}, \mathrm{NA} / 0.25)$ and provides a cross-sectional image over $0.6 \mathrm{~mm}$ of depth without focus tracking, thus holding great promise of endoscopic optical biopsy for diagnosis and grading of flat epithelial cancer such as carcinoma in situ in vivo. $\odot 2007$ Society of Photo-Optical Instrumentation Engineers. [DOI: 10.1117/1.2800007]

Keywords: tomography; optics; coherence; biomedical optics; subcellular; cancer.

Paper 07168LR received May 9, 2007; revised manuscript received Aug. 7, 2007; accepted for publication Aug. 22, 2007; published online Oct. 24, 2007.

Noninvasive in vivo imaging identification of pathogenesis at cellular resolutions is crucial to early clinical diagnosis of cancers. ${ }^{1}$ Technological advance in confocal microscopy and endoscopy has permitted noninvasive imaging of cellular morphology in intact tissue such as skin and bladder, colon, and cervical epthelia, but imaging depth is limited to 100 to $300 \mu \mathrm{m}$ and focus-tracking is required, which is difficult in many clinical applications. Unlike confocal microscopy, the lateral and axial resolutions of optical coherence tomography (OCT) are decoupled, ${ }^{2}$ e.g., the axial resolution is determined by the source coherence length $L_{c}=2 \ln 2 / \pi \lambda^{2} / \Delta \lambda$, where $\lambda$ and $\Delta \lambda$ are the source central wavelength and full-width half maximum (FWHM) spectral bandwidth. Therefore, the axial sectioning resolution of OCT can be substantially improved by employing broadband sources, and subcellular imaging of low-scattering tissue such as xenopus laevis (mesenchymal cells) in vivo has been reported. ${ }^{1,2}$ However, for mammalian epithelial cells, the smaller cell size and thus denser micro-

*Tel: (631) 444-1451; E-mail: yingtian.pan@sunysb.edu

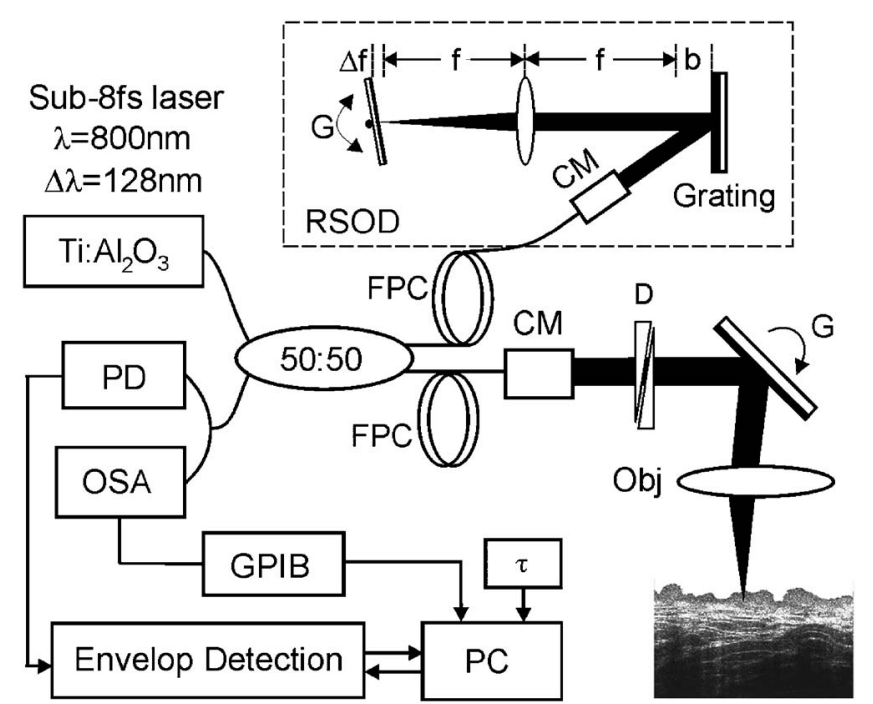

Fig. 1 A schematic of the time-domain uOCT setup. CM: fiberoptic collimator, PD: photodiode, FPC: polarization controller, D: BK7 wedge prism pair; G: servo mirror, OSA: optical spectrum analyzer, RSOD: grating-lens-based rapid optical delay, Obj: achromatic lens, $\tau$. trigger delay for time-lapse uOCT. Spectral reshaping was implemented by adjusting the chromatic aberration (i.e., $\Delta f$ ) in the RSOD and optimizing $S_{s r}(\lambda)$ and $\Delta z$ with the OSA instantaneously.

organelle content results in increased cellular scattering, leading to phase randomization, i.e., speckle noise, that may drastically degrade the image contrast and resolution for imaging subcellular details. For instance, ultrahigh-resolution OCT (uOCT) with axial resolution exceeding $0.7 \mu \mathrm{m}$ has been reported, ${ }^{2}$ but imaging of subcellular details of the epithelium remains unsolved, preventing this promising technique from becoming an optical biopsy tool for clinical diagnosis. In this letter, we demonstrate that the subcellular details of living rat urothelium can be uncovered after proper time-lapse dynamic averaging to minimize speckle noise in uOCT imaging.

Figure 1 is a schematic of the time-domain uOCT setup used in this study. A Ti:Sapphire laser $(\Delta \lambda=128 \mathrm{~nm})$ was used to illuminate a wavelength-flattened broadband fiberoptic Michelson interferometer. In the sample arm, light was collimated to $\phi 4.7 \mathrm{~mm}$ by a fiberoptic achromatic lens, scanned laterally by a $\phi 8$-mm servo mirror, and focused onto biological tissue under examination by a commercial-grade achromatic lens (f/10 mm, NA/0.25) to yield a focal spot size (lateral resolution) of $\Delta r \approx 3 \mu \mathrm{m}$ based on ZEMAX modeling. In the reference arm, light from the fiber was collimated to $\phi 2 \mathrm{~mm}$, attenuated and connected to a rapid scanning optical delay (RSOD, $d^{-1}=1200 \mathrm{~mm}, f=80 \mathrm{~mm}$ ) for axial scanning at a stable Doppler frequency shift $\left(f_{D}=800 \mathrm{kHz}\right)$ for heterodyne detection. ${ }^{3}$ Then, the light beams from both the sample and reference arms were recombined in the detection fiber, and the detected interferometric signal was bandpassfiltered at $f_{D}$, the amplitude was linearly demodulated, and the signal was digitized via a $5 \mathrm{MHz}, 12$ bit A/D for twodimensional (2-D, up to $8 \mathrm{fps}$ ) and three-dimensional (3-D) uOCT image reconstruction. Dispersion compensation and ultra-broadband propagation were crucial for uOCT imaging and were implemented separately. Mismatch of dispersions, 


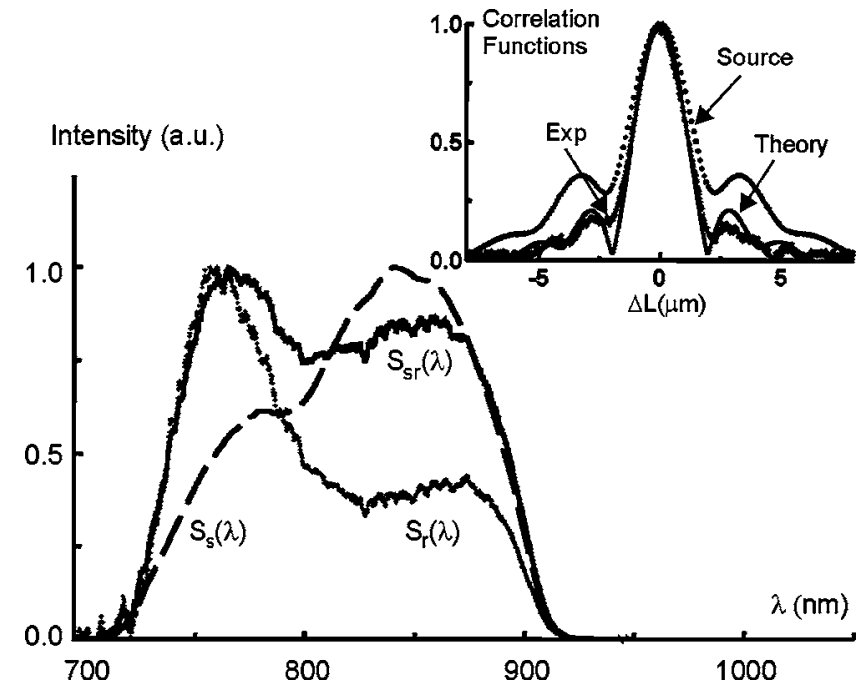

Fig. 2 Measured power spectra $S_{s}(\lambda), S_{r}(\lambda)$ and $S_{s r}(\lambda)$ and crosscorrelation functions. $\Delta \lambda_{s}=113 \mathrm{~nm}$ and $\Delta \lambda_{r}=62 \mathrm{~nm}$, but spectral shaping allowed $\Delta \lambda_{s r}=155 \mathrm{~nm}>\Delta \lambda=128 \mathrm{~nm}$. The inset shows that the axial resolution $\Delta z=2.39 \mu \mathrm{m}$ reached the transform limit $(\Delta z$ $=2.34 \mu \mathrm{m})$ and exceeded the source $L_{c}=2.95 \mu \mathrm{m}$.

e.g., group velocity dispersion (GVD) and third-order dispersion (TOD), between the sample and reference arms was compensated $^{3}$ by RSOD (adjusting $b$ ) in combination with a pair of adjustable BK7 wedge prisms ${ }^{2}$ (adjusting prism depth $D)$. Chromatic aberration (mostly from Obj and RSOD) was found to be a major technical challenge that led to drastically reduced effective spectral bandwidth and thus axial resolution. ${ }^{2}$ We tackled this problem by maximizing the detected bandwidth of the cross spectrum $S_{s r}(\lambda)$ $=\left[S_{S}(\lambda) \cdot S_{r}(\lambda)\right]^{1 / 2}$, and we employed an optical spectrum analyzer interfaced with a PC to monitor the changes in $S_{s r}(\lambda)$ and the cross-correlation function whose FWHM width defines the transform-limited axial resolution $\Delta z$ in near real time while optimizing chromatic aberrations in both arms (mostly by adjusting $\Delta f$ in RSOD). Figure 2 shows the traces of the measured power spectra and the correlation functions. The results indicate that due to uncorrected chromatic aberrations, both $S_{s}(\lambda)$ and $S_{r}(\lambda)$ were narrower than $S_{i}(\lambda)$, e.g., $S_{s}(\lambda)$ peaked at $\lambda>800 \mathrm{~nm}$. However, $S_{r}(\lambda)$ could be easily adjusted by RSOD to peak at $\lambda<800 \mathrm{~nm}$ so that $S_{s r}(\lambda)$ could be broadened to $\Delta \lambda=155 \mathrm{~nm}$, which exceeded the source $S_{i}(\lambda)$ bandwidth $\Delta \lambda=128 \mathrm{~nm}$. As a consequence, the measured axial resolution was narrower than the source coherence length, i.e., $\Delta z=2.39 \mu \mathrm{m}<L_{c}=2.95 \mu \mathrm{m}$. Moreover, the two major lobes with over $38 \%$ of peak power were reduced to $<18 \%$ due to improved spectral shape. It was found that $\Delta z<2.5 \mu \mathrm{m}(\sim 1.8 \mu \mathrm{m}$ in epithelium, assuming the refractive index of biological tissue $n=1.38$ ) with low sidelobes was crucial to time-lapse uOCT delineation of epithelial nuclei $(\sim \phi 7 \mu \mathrm{m})$.

Studies have revealed that although uOCT with an axial resolution of $1.8 \mu \mathrm{m}$ or less is able to clearly delineate the subcellular details of xenopus laevis, ${ }^{1,2}$ subcellular uOCT of scattering tissue such as mammalian epithelium-critical to cancer diagnosis-remains unsolved. To exclude the influence of relatively lower lateral resolution $\Delta r \approx 3 \mu \mathrm{m}$ than that of optical coherence microscopy (OCM), we performed uOCT imaging of rat bladder epithelium by replacing the $\mathrm{f} / 10 \mathrm{~mm}$ achromat (Obj) with a fully corrected microscopic objective (e.g., Nikon 16X/0.8NA water lens), but experiments concluded that no subcellular visualization although both axial and lateral resolutions were optimized, i.e., $\Delta z \approx 1.8 \mu \mathrm{m}$ and $\Delta r \approx 0.7 \mu \mathrm{m}$, to be sufficient to resolve the epithelial membrane and nucleus. This result suggested that uOCT as an interference-based imaging technique is different from an intensity-based approach such as confocal microscopy, ${ }^{4,5}$ whose spatial resolution is no longer determined by the focusing spot size (e.g., ultimately under the diffraction limit) but rather by speckle noise due to phase randomization among the local interfering scattering waves that may lead to severely degraded image contrast and reduced spatial resolution. In other words, the subcellular details may be uncovered if the speckle effects can be effectively reduced. For proof of concept, we applied various speckle reduction techniques, e.g., A-scan, frame, and composite NA averaging. ${ }^{6}$ We found that proper time-lapse frame averaging was a simple and effective approach that took advantage of dynamic micro motion in living biological tissue such as urinary bladder. Figure 3 shows an example of a fresh living rat bladder ex vivo. Panel (a) is a snapshot showing no visible cellular morphology in the urothelium due to speckle noise, whereas panel (b) is an average over four frames with optimized time lapse $(\tau$ $\approx 0.4 \mathrm{~s}$ ). It must be noted that the time lapse was crucial: If $\tau$ was too short, the motion-induced phase scrambling was insufficient to reduce speckle noise; if $\tau$ was too long, the motion artifact washed away the subcellular details. $\tau \approx 0.4 \mathrm{~s}$ was determined experimentally based on spontaneous motion of the living bladder tissue under examination. As shown in Fig. 3, the difference was incredible-the seemingly impossible nuclear morphology $(N)$ of living epithelial cells was clearly uncovered. In contrast to the low-scattering large mesenchymal cells whose nuclei and cell membranes appeared highly backscattered in the uOCT image, ${ }^{1,2}$ the membranes of the epithelial cells were submerged in highly backscattering cytoplasm but the epithelial nuclei appeared low backscattering, in agreement with OCM observations. ${ }^{4,5}$ The fact of "dark" nuclei embedded in highly backscattering cytoplasm supported our hypothesis that speckles resulting from highly intracellular scattering (due to denser cytoplasm than in mesenchymal cells) ruined the subcellular details in an uOCT image of mammalian epithelial cells. According to Mie's theory of scattering, small micro-organelles contribute favorably to backscattering (i.e., uOCT signal), whereas large nuclei favor forward scattering. The nuclear size of rat epithelial cells measured by uOCT, $D_{N}=(7.9 \pm 1.4) \mu \mathrm{m}$, compared well to that of the corresponding histology in panel (c), $D_{N}$ $=(7.2 \pm 0.8) \mu \mathrm{m}$. More importantly, morphological details of the rat bladder at depths of $600 \mu \mathrm{m}$ below the bladder surface, including urothelium, lamina proporia, and upper muscularis, were well delineated without focus tracking and image fusion. Calibration of uOCT signal drop (using mirror reflectance) along the $z$ direction revealed that the depth of focus was $\sim 120 \mu \mathrm{m}$; nevertheless, the high scattering in lamina proporia and muscularis still allowed delineation of bladder structures down to $600 \mu \mathrm{m}$ below the urothelium at which the focus was centered.

In summary, we present the first experimental evidence of subcellular uOCT imaging of the epithelium. By implement- 


\section{JBO LETTERS}
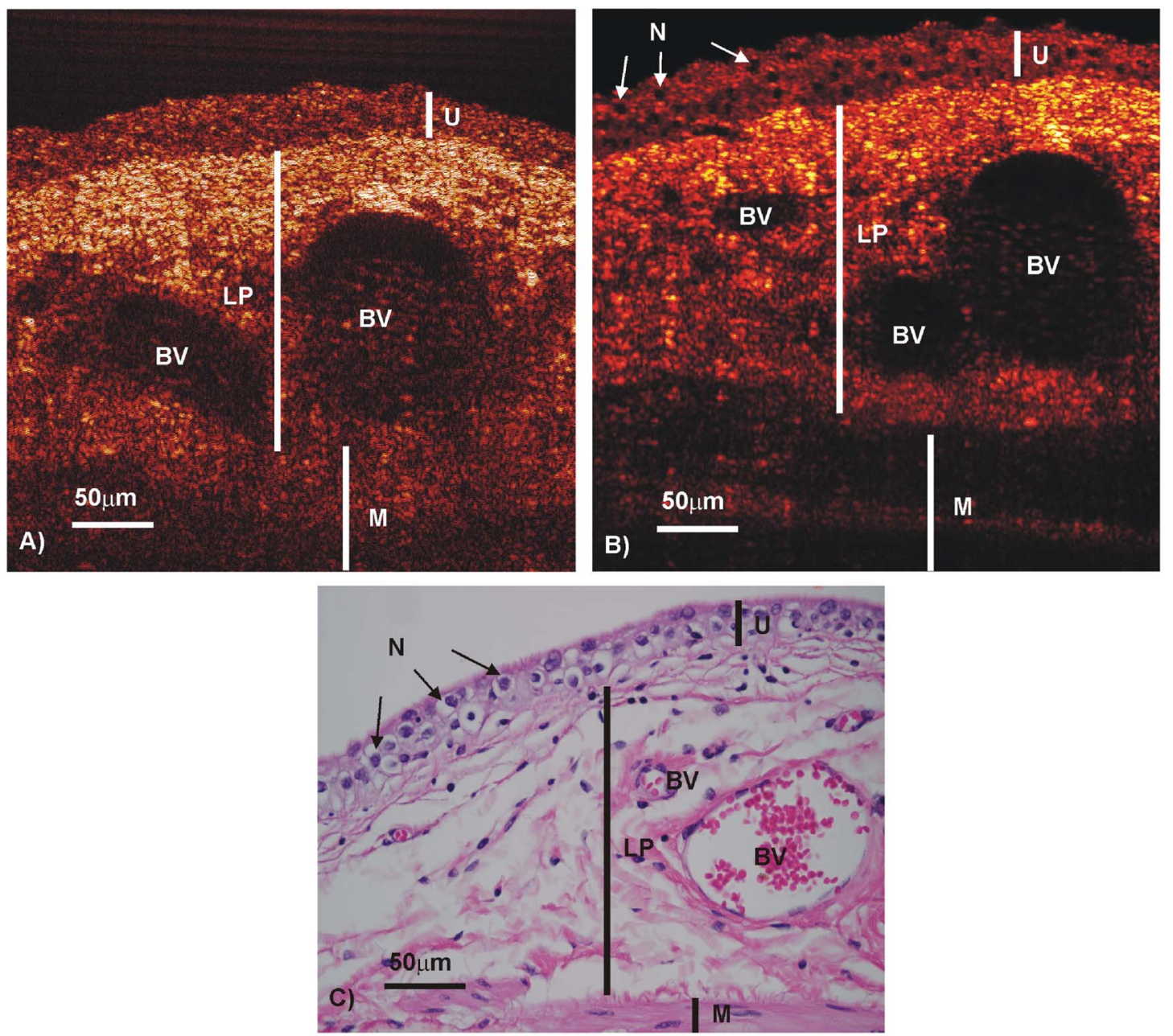

Fig. 3 Subcellular uOCT of living rat bladder ex vivo using a f/10 mm achromat (NA $\approx 0.25)$. (a) A snapshot, (b) time-lapse dynamic frame averaged image, and (c) corresponding histology of (b). U: urothelium, LP: lamina propria, M: upper muscularis, BV: bladder vessel, N: urothelial nucleus.

ing time-lapse dynamic frame averaging that took advantage of the micromotion of living tissue to effectively reduce speckle noise, the nuclei of the rat bladder epithelium were uncovered and confirmed by the corresponding histology. Unlike OCM, ${ }^{3,5}$ which requires a sophisticated high-NA microscopic objective, the reported approach used a single achromat $(\mathrm{f} / 10 \mathrm{~mm}, \mathrm{NA} \approx 0.25)$ and provided a cross-sectional image over depths of $0.6 \mathrm{~mm}$ without focus tracking, thus holding great promise for optical biopsy to diagnose and grade flat epithelial cancers that require noninvasive in vivo imaging at subcellular resolutions. Testing of a microelectromechanical systems uOCT endoscope based on dynamic frame averaging is being conducted for in vivo subcellular imaging of bladder epithelium. It was found that $\Delta z$ $<2.5 \mu \mathrm{m}$ (exceeding the source transform limit of $L_{c}$ $=2.95 \mu \mathrm{m}$ ) was important for time-lapse uOCT to segment the boundaries and thus to estimate the nuclear sizes; however, further experimentation is needed to determine the threshold of spatial resolution required to resolve $\sim \phi 7 \mu \mathrm{m}$ epithelial nuclei. It was also noticed in Fig. 3 that uOCT tended to delineate fewer nuclei than the corresponding histology and slightly overestimated the nuclear sizes $(<10 \%)$, which could be the potential limitation of time-lapse dynamic frame averaging; nevertheless, this does not prevent this promising technique from grading epithelial cancers, based on our recent rodent bladder cancer study.

This work was supported in part by NIH Grant Nos. 2R01DK059265 and R01-DK068401.

\section{References}

1. S. A. Boppart, B. E. Bouma, C. Pitris, J. F. Southern, M. E. Brezinski, and J. G. Fujimoto, "In vivo cellular optical coherence tomography imaging," Nat. Med. 4, 861-865 (1998).

2. W. Drexler, "Ultrahigh-resolution optical coherence tomography," $J$. Biomed. Opt. 9, 47-74 (2004).

3. T. Q. Xie, Z. G. Wang, and Y. T. Pan, "Dispersion compensation in high-speed optical coherence tomography using acousto-optic modulation," Appl. Opt. 44, 4272-4280 (2005).

4. E. Beaurepaire, L. Moreaux, F. Amblard, and J. Mertz, "Combined scanning optical coherence and two-photon-excited fluorescence microscopy," Opt. Lett. 24, 969-971 (1999).

5. S. Tang, C.-H. Sun, T. B. Krasieva, Z. P. Chen, and B. J. Tromberg, "Imaging subcellular scattering contrast by using combined optical coherence and multiphoton microscopy," Opt. Lett. 32, 503-505 (2007).

6. J. M. Schmitt, "Array detection for speckle reduction in optical coherence microscopy," Phys. Med. Biol. 42, 1427-1439 (1997). 